\title{
Decreased FOXFI promotes hepatocellular carcinoma tumorigenesis, invasion, and stemness and is associated with poor clinical outcome
}

This article was published in the following Dove Press journal:

OncoTargets and Therapy

23 March 2016

Number of times this article has been viewed

\author{
Zhen-guo Zhao',2 \\ De-qiang Wang ${ }^{3}$ \\ De-fei $\mathrm{Hu}^{4}$ \\ You-sheng $\mathrm{Li}^{1}$ \\ Shuang-hai Liu²
}

'Department of Surgery, Jinling Hospital, Nanjing Medical University, Nanjing, ${ }^{2}$ Department of General Surgery, The Affiliated Jiangyin Hospital of Southeast University Medical College, Jiangyin, ${ }^{3}$ Tumor Treatment Center, The Affiliated Hospital of Jiangsu University, Zhenjiang, ${ }^{4}$ Clinical Laboratory, The Second People's Hospital of Huai'an, Huai'an, People's Republic of China

Correspondence: De-fei Hu

Clinical Laboratory, The Second People's Hospital of Huai'an, Huaihai

Road, Huai'an, Jiangsu 223002, People's

Republic of China

Tel +86 5 I7 $8087 \quad$ I636

Email drhudefei@I63.com

You-sheng Li

Department of Surgery, Jinling

Hospital, Nanjing Medical University,

Nanjing 210066, People's Republic

of China

Tel +86258086 0II4

Email weicuibeifang@sina.com

\begin{abstract}
Forkhead box F1 (FOXF1), a member of the forkhead transcription factor superfamily, plays critical roles in the progression of certain types of cancers. However, the expression and function of FOXF1 in human hepatocellular carcinoma (HCC) are still unclear. Quantitative real-time reverse transcription polymerase chain reaction, Western blotting, and immunohistochemistry detected the relatively lower expression status of FOXF1 in HCC cases. Soft agar and transwell assays clearly demonstrated that FOXF1-knockdown cells showed significantly increased in vitro cell tumorigenesis and invasion, and FOXF1-overexpressing cells had significantly reduced growth and invasion potential. Our study also examined the role of FOXF1 in HCC cell stemness by sphere formation, aldehyde dehydrogenase (ALDH1) activity, and CD44/133-positive cell analysis. Enforced FOXF1 expression decreased HCC cell stemness, and the downregulation of FOXF1 promoted cancer cell stemness. The in vivo study showed that overexpressed FOXF1 inhibits nude mouse tumorigenicity with downregulation of CD44 and proliferating cell nuclear antigen. More importantly, loss of FOXF1 expression was linked to poor overall survival time by Kaplan-Meier analysis.
\end{abstract}

Keywords: hepatocellular carcinoma, FOXF1, tumorigenesis, invasion, cancer stemness, clinical outcome

\section{Introduction}

Liver cancer is the fifth most common cancer worldwide and the second most frequent cause of cancer death in men. An estimated 748,300 new cases of liver cancer and 695,900 cancer-associated deaths occurred worldwide in 2008. ${ }^{1}$ Hepatocellular carcinoma (HCC) is a major concern in liver cancer, as the mechanisms underlying its development remain unclear and the therapeutic outcome is poor. ${ }^{2-4}$ Surgical resection is the most common and effective treatment for early-stage patients, but the recurrent rate is as high as $50 \%$ within 2 years. ${ }^{5}$ Intra- and extrahepatic metastases occur in more than half of HCC patients after resection. ${ }^{6}$ Therefore, investigation of the molecular pathogenesis of $\mathrm{HCC}$ and identification of new potential targets for preventing the progression of HCC are imperative.

Forkhead box (FOX) proteins comprise a family of evolutionarily DNA-binding transcriptional regulators that play important roles in both biological processes and cancer development. ${ }^{7-9}$ Expression levels of the FOX transcription factors determine the development and progression of diseases. ${ }^{7,9}$ The FOXF1 protein is a member of this superfamily and regulates cellular proliferation, differentiation, and metabolic homeostasis. ${ }^{10}$ In addition to its indispensable functions in organ morphogenesis, ${ }^{11-13}$ human FOXF1 is also linked to tumorigenesis. In lung cancer, FOXF1 regulates 
cancer-associated fibroblasts to stimulate cancer cell migration and tumor growth. ${ }^{14}$ Furthermore, significantly higher expression levels of FOXF1 were found in non-small-cell lung cancer cases with lymph node metastasis. ${ }^{15}$ Overexpression of FOXF1 in a mouse mammary epithelial cell line was associated with epithelial-mesothelial transition phenotype and invasive potential. ${ }^{16}$ In addition to these oncogenic roles of FOXF1, reduced expression of FOXF1 in breast cancer was shown to be due to epigenetic silencing, and FOXF1 reexpression inhibited growth and tumorigenicity of breast cancer cell lines mainly by the blocking of G1-S cell cycle progression. ${ }^{17}$ A study in colorectal adenocarcinomas showed that the poor prognosis of colorectal cancer is associated with cytoplasmic mislocalization of overexpressed FOXF1. ${ }^{18}$

Cancer stem cells are a subset of cancer cells with the ability for self-renewal and differentiation. The regulation of the stem cell-like properties by several important signaling pathways has been found to endow the HCC cells with an increased level of tumorigenicity and metastatic ability. ${ }^{19}$ In endometrial cancer, FOXO1, another forkhead transcription factor superfamily protein, has been proven to inhibit CD44 expression through Wnt/ $\beta$-catenin. ${ }^{20}$ Whether FOXF1 contributes to HCC cancer stemness is still unknown.

Because little is known about the role of FOXF1 in HCC, we assessed the role of FOXF1 on the regulation of HCC cell tumorigenesis, invasion, and stemness. Moreover, the characterization of the underlying molecular events, which may in turn provide a molecular basis for applying FOXF1 as a novel target for the clinical treatment of HCC, was also attempted.

\section{Materials and methods}

\section{Patients and tissue specimens}

One hundred and forty-three pairs of human tissue specimens containing $\mathrm{HCC}$ tissues and noncancerous adjacent tissues of HCC patients undergoing curative resection were obtained from the Affiliated Jiangyin Hospital of Southeast University Medical College with written informed consent and ethical approval was obtained from the Ethics Committee of the same hospital (No::20130032X2). No patient underwent radiotherapy or chemotherapy prior to surgery, and clinical data were collected from all patients.

\section{Immunohistochemistry and evaluation}

Immunohistochemistry was performed using formalin-fixed, paraffin-embedded tissue sections. The sections were deparaffinized using a graded ethanol series and then incubated in $3 \%$ hydrogen peroxide for 10 minutes to block endogenous peroxidase. Primary antibody for human FOXF1 (1:150 dilution; Chemicon, Billerica, MA, USA) was applied overnight at $4^{\circ} \mathrm{C}$. The procedure followed was the streptavidinperoxidase method (ZS-BIO, Beijing, People's Republic of China), and the sections were developed with diaminobenzidine chromogen (Boster, Wuhan, People's Republic of China). Finally, immunoreactivity was scored as the nuclear staining intensity (on a scale of $0-3$ : negative $=0$, weak $=1$, moderate $=2$, strong $=3$ ) $\times$ the percentage of cells stained (positively recorded on an ordered categorical scale: score of $0=0 \%, 1=1 \%-25 \%, 2=26 \%-50 \%$, and $3=51 \%-100 \%$ ), which resulted in a scale of $0-9$. Immunoreactivity was evaluated separately by two senior pathologists. Any discrepancy between the two evaluators was resolved by reevaluation and careful discussion until agreement was reached.

\section{RNA extraction and real-time reverse transcription polymerase chain reaction analysis}

Total RNA was isolated with ice-cold TRIZOL reagent (Thermo Fisher Scientific, Waltham, MA, USA), then used as the template for reverse transcription for complementary DNA (cDNA) synthesis. Quantitative real-time reverse transcription polymerase chain reaction (Q-RTPCR) was used to detect the expression level of FOXF1. Q-RTPCR was performed on ABI StepOne Sequence Detection System using the SYBR ${ }^{\circledR}$ Green (TaKaRa Biotechnology Co Ltd, Dalian, People's Republic of China) detection method in accordance with the manufacturer's instructions under the following conditions: $95^{\circ} \mathrm{C}$ for 10 minutes, and 40 cycles of $95^{\circ} \mathrm{C}$ for 5 seconds and $55^{\circ} \mathrm{C}$ for 30 seconds. Expression levels of the analyzed genes were normalized to the expression of $\beta$-actin. The sequences of the primer pairs were as follows: FOXF1 forward: 5'-CACTCCCTGGAGCAGCCGTATC-3' and reverse: $5^{\prime}$-AAGGCTTGATGTCTTGGTAGGTGA-3'; CD44 forward: 5'-CACGTGGAATACACCTGCAA-3' and reverse: 5'-GACAAGTTTTGGTGGCACG-3'; CD133 forward: 5'-TTTGGATTCATATGCCTTCTGT-3' and reverse: 5'-CCATTGGCATTCTCTTTGAA-3'; $\beta$-actin forward: 5'-ATAGCACAGCCTGGATAGCAACGTAC-3' and reverse: 5'-CACCTTCTACAATGAGCTGCGTGTG-3'. Comparative cycle threshold $(\mathrm{Ct})$ method was used for quantification of the transcripts.

\section{Construction of the FOXFI expression plasmid and small interfering RNA}

To construct a $F O X F 1$-expressing plasmid, full-length cDNA fragments of the FOXF1 gene were obtained by RTPCR of RNA and cloned into the pGEM-T-Easy vector (Promega 
Corporation, Fitchburg, WI, USA). Positive clones were selected, and then the FOXF1 cDNA sequence was subcloned into the pcDNA3.1 (-) vector (Thermo Fisher Scientific) with the hemagglutinin tag coding sequence.

Human FOXF1 small interfering RNA (siRNA) and one negative control siRNA were synthesized by RiboBio Co, Ltd (Guangzhou, People's Republic of China). The targeted sequences of the human FOXF1 siRNA were 5'-GCTCA ACGAGTGCTTCATCAAGCTACCCA-3' (siFOXF1-1); 5' - T G T G T GA C C GA A A GGA G T T T G T C T T - 3' (siFOXF1-2).

\section{Cell culture and transfection}

Five human HCC cell lines HepG2, SMMC-7221,SNU-182, Hep3B, and SNU-449 were obtained from the Cell Bank of Type Culture Collection of Chinese Academy of Sciences (Shanghai, People's Republic of China), and were cultured in Roswell Park Memorial Institute 1640 medium (Thermo Fisher Scientific) containing 10\% fetal bovine serum (FBS) (Thermo Fisher Scientific) at $37^{\circ} \mathrm{C}$ with $5 \% \mathrm{CO}_{2}$.

To get a stable FOXF1-overexpressing cell line, the FOXF1 expression plasmid pCDNA3.1-FOXF1 was transfected into SNU-182 cells, followed by selection on $800 \mu \mathrm{g} / \mathrm{mL}$ of neomycin for 21 days. FOXF1-specific siRNA was transfected into HepG 2 cells. All the transfection experiments used Lipofectamine 2000 (Thermo Fisher Scientific), and the cells were incubated according to the manufacturer's instruction.

\section{Western blotting assay}

Cells were lysed in ice-cold radio immunoprecipitation assay lysis buffer containing protease inhibitor cocktails. Equivalent amounts of proteins $(20 \mu \mathrm{g})$ were then separated by $10 \%$ sodium dodecyl sulfate-polyacrylamide gel electrophoresis and transferred to polyvinylidine difluoride membranes (EMD Millipore, Billerica, MA, USA). After blocking, primary antibodies against FOXF1 (1:1,000; Chemicon), matrix metalloproteinase-2 (MMP2) (1:1,000; Cell Signaling Technology, Danvers, MA, USA), proliferating cell nuclear antigen (PCNA) (1:1,000; Abcam), CD44 (1:1,000; Cell Signaling Technology), and $\beta$-actin $(1: 2,000$; Santa Cruz Biotechnology Inc., Dallas, TX, USA) were separately incubated with the membranes at $4^{\circ} \mathrm{C}$ overnight. Then, secondary antibodies were added and the membranes were incubated at room temperature for 2 hours. Protein bands were detected on a G:Box iChem Imager (Syngene, Cambridge, UK) using enhanced chemiluminescence (Pierce; Thermo Fisher Scientific).

\section{Soft agar assay}

One thousand HCC cells were suspended as a single-cell suspension in $0.35 \%$ agarose in RPMI 1640 containing $10 \% \mathrm{FBS}$ in $0.7 \%$ agarose in the same medium in six-well plates. After 21 days' incubation, the colonies were stained with $0.5 \%$ crystal violet. Only colonies with $>50$ cells were counted. Each treatment was repeated in triplicate.

\section{Transwell invasion assay}

Cell invasion assay was performed in a 24-well Transwell chamber with a pore size of $8 \mathrm{~mm}$ (Costar, New York, NY,

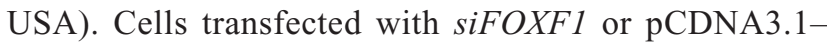
FOXF1 were seeded into the insert chambers that were coated with Matrigel (BD Biosciences, San Jose, CA, USA) in serum-free medium. Medium containing $10 \%$ FBS was added to the lower chamber as a chemoattractant. After incubation for 24 hours, the invaded cells were fixed with $4 \%$ paraformaldehyde, stained with $0.1 \%$ crystal violet, and quantified from five microscopic fields.

\section{Sphere formation assay}

Cells were trypsinized and washed with phosphate-buffered saline (PBS) twice. Next, $1.0 \times 10^{3}$ cells were seeded in the wells of six-well ultralow attachment plates (Corning Incorporated, New York, NY, USA) in Dulbecco's Modified Eagle's Medium/F-12 culture medium supplemented with $10 \mathrm{ng} / \mathrm{mL}$ human recombinant basic fibroblast growth factor (PeproTech Inc, Rocky Hill, NJ, USA) and 10 ng/mL epidermal growth factor (PeproTech). After culturing for 14 days, spheres were photographed and counted.

\section{Flow cytometry analysis}

The Aldefluor Kit (Stemcell Technologies, Vancouver, BC, Canada) was used to identify cell populations possessing aldehyde dehydrogenase (ALDH1) activity. The assay was carried out according to manufacturer's instructions. Control cells were stained using identical conditions but included a specific ALDH inhibitor, diethylaminobenzaldehyde, to serve as a negative control for each experiment. Such cells are recognized by comparing the fluorescence in a test sample to that in a control sample containing diethylaminobenzaldehyde. As only cells with an intact cellular membrane can retain the Aldefluor reaction product, only viable ALDH1-positive cells are identified. The brightly fluorescent ALDH1-expressing cells (ALDH1-positive) were detected using a BD FACScan flow cytometer (Becton Dickinson [BD], Franklin Lakes, NJ, USA) and calculated as the percentage of ALDH1-positive cells in each cell line. 
CD44/133-positive cells were analyzed by flow cytometry. Briefly, $1 \times 10^{6}$ cultured cells were incubated with $100 \mu \mathrm{L}$ of $1 \%$ bovine serum albumin in PBS containing $1 \mu \mathrm{g}$ of CD16/32 (eBioscience, San Diego, CA, USA) for 30 minutes on ice to block nonspecific fragment crystallizable $(\mathrm{Fc})$ interaction, then the cells were labeled with phycoerythrinconjugated anti-CD133 and fluorescein isothiocyanateconjugated anti-CD44. Isotypic IgG and unstained cells served as negative controls.

\section{In vivo tumor xenograft assay}

All animal experiments were approved by the Animal Care and Use Committee of the Affiliated Jiangyin Hospital of Southeast University Medical College. The cells were resuspended in $40 \%$ Matrigel matrix (BD Biosciences) in PBS at a density of $1 \times 10^{7}$ cells $/ \mathrm{mL}$. Six-week-old male athymic mice were housed in a specific pathogen-free facility. Stable FOXF1-overexpressing SNU-182 cells and control SNU-182 cells were injected subcutaneously into the flank region of nude mice using a $1-3 \mathrm{~cm}^{3}$ tuberculin syringe. Tumor size was measured every 7 days using a Vernier caliper, and tumor volume was calculated using the following equation: tumor volume $\left(\mathrm{mm}^{3}\right)=\left(\mathrm{L} \times \mathrm{W}^{2}\right) / 2$, where $\mathrm{L}$ is the length and $\mathrm{W}$ is the width. At the end point, the tumors were harvested and weighed.

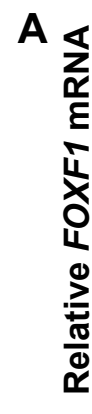

C

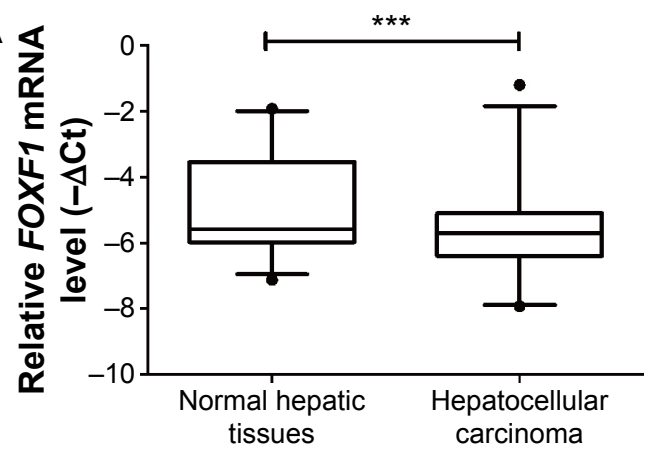

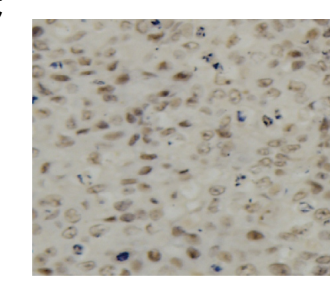

Normal hepatic tissues

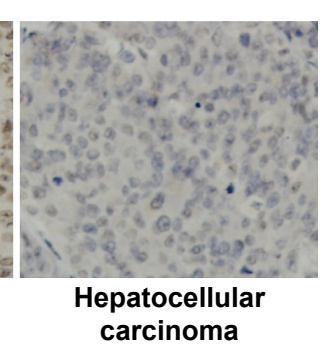

\section{Statistical analyses}

Statistical analyses were performed using SPSS16.0 software (SPSS Inc., Chicago, IL, USA). All data were presented as mean \pm standard error (SE). For the relationship between $F O X F 1$ expression and clinicopathologic features, $P$-values were calculated using the $\chi^{2}$ test. The differences between two groups were analyzed with Student's $t$-test. $P<0.05$ was considered statistically significant.

\section{Results \\ FOXFI expression in HCC}

We used Q-RTPCR to evaluate the mRNA expression of FOXF1 in 30 pairs of HCC and noncancerous adjacent tissues. Reduced FOXF1 expression levels were detected in HCC tissues compared with noncancerous tissues (Figure 1A), which was consistent with immunohistochemical data. Western blotting confirmed similar results in four pairs of $\mathrm{HCC}$ and noncancerous adjacent tissues (Figure 1B). FOXF1 staining status is shown in Figure 1C. Among 143 HCC tissue specimens and their matched noncancerous adjacent tissues, the immunohistochemical indexes of FOXF1 were $2.368 \pm 0.3297$ for HCC tissues compared with $4.061 \pm 0.1678$ for noncancerous adjacent tissues (Figure 1D), indicating that FOXF1 expression was significantly decreased in HCC compared with noncancerous tissues $(P<0.0001)$.

\section{B}

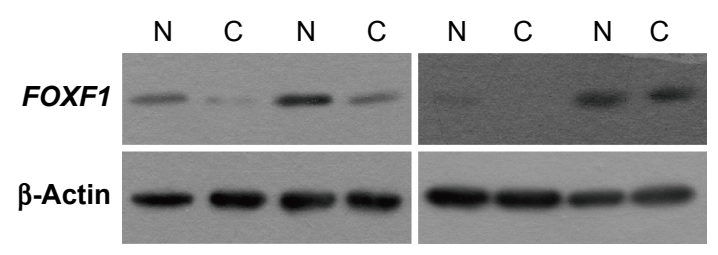

D

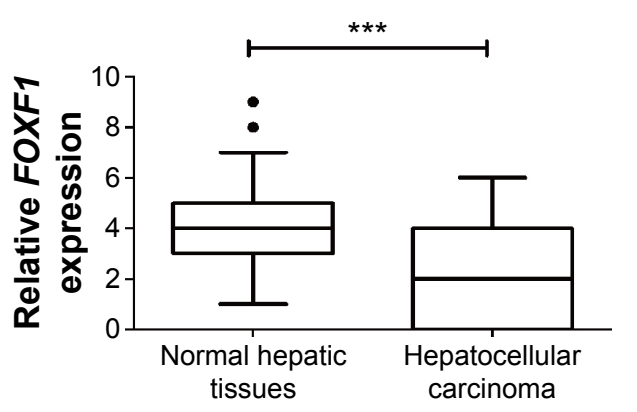

Figure I Expression of FOXFI in HCC and noncancerous adjacent tissues.

Notes: (A) Quantitative RTPCR evaluation of FOXFI expression on mRNA levels in 30 pairs of collected HCC tissues and adjacent normal tissues. (B) Representative Western blot of FOXFI protein levels in HCC tissues (indicated by the letter C) and adjacent normal tissues (indicated by the letter N). (C) Representative FOXFI staining in $\mathrm{HCC}$ specimens, FOXFI have high expression in normal hepatic tissue nuclear and decreased in HCC nuclear. (D) Box-whisker plots presenting the mean \pm SEM value of FOXFI staining values in 143 pairs of tissues. ${ }^{* * *}<<0.00$ I, bar scale $50 \mu \mathrm{m}$. The filled circles in $\mathbf{A}$ and $\mathbf{D}$ are the values out of confidence interval (5\%-95\%).

Abbreviations: FOXFI, forkhead box FI; HCC, hepatocellular carcinoma; RTPCR, reverse transcription polymerase chain reaction; SEM, standard error of the mean; $\mathrm{Ct}$, cycle threshold. 


\section{FOXFI expression in HCC cell lines and its effects on tumorigenesis and invasion in vitro}

We next investigated the levels of FOXF1 expression in different HCC cell lines, including HepG2, SNU-182, Hep3B, SNU-449, and SMMC-7221 cells. Results showed that FOXF1 level was much higher in HepG2 cells than in the other four cell lines and was lowest in SNU-182 cells (Figure 2A). Therefore, we used HepG2 cells and SNU-182 cells for further experiments.

When FOXF1 was knocked down in HepG2 cells by RNA interference, the growth-related molecule, PCNA, and the metastasis-related molecule, MMP2, showed upregulation. In FOXF1-overexpressing stable SNU-182, cells had decreased PCNA and MMP2 protein levels (Figure 2B). Soft agar assay showed that overexpression of FOXF1 significantly inhibited the in vitro tumorigenesis of SNU-182 cells $(P=0.0153$; Figure $2 \mathrm{C})$. In contrast, siRNA silencing of FOXF1 in HepG2 cells showed a significant increase in cell growth compared with the control group (siFOXF1-1, $P=0.0057$; siFOXF1-2, $P=0.0150$; Figure 2C). We also evaluated the invasion abilities of FOXF1-knockdown HepG2 cells and FOXF1-overexpressing SNU-182 cells using Matrigel invasion assays. We found that FOXF1-knockdown HepG2 cells showed a significant increase in invasion ability compared with control cells (siFOXF1-1, $P=0.0012$; siFOXF1-2, $P=0.0042$; Figure 2D), while forced FOXF1 expression significantly reduced invasion of SNU-182 cells compared with control cells $(P=0.0064$; Figure 2D). Together, these data indicated that FOXF1 affects HCC cell proliferation and invasion.

\section{FOXFI regulates HCC stemness}

HCC cell stemness was previously found to affect cancer cell proliferation, invasion, and tumorigenesis. ${ }^{19}$ To identify whether FOXF1 expression correlates with HCC cell stemness, the mRNA levels of FOXF1, CD44, and $C D 133$ were detected by Q-RTPCR, and the comparisons $F O X F 1$ vs $C D 44$ ( $P=0.0049)$ and $F O X F 1$ vs $C D 133(P=0.0015)$ were found to have significant negative correlation (Figure $3 \mathrm{~A}$ ). To identify whether FOXF1 affects HCC cell stemness, sphere formation assay, ALDH1 activity, and CD44/133-positive cell counting were used to validate the hypothesis. As shown in Figure 3B-D, decreased level of $F O X F 1$ in Hep G2 promotes cancer cell sphere formation ability as well as ALDH1- and CD44/133-positive cell number, whereas the overexpression of FOXF1 inhibits SNU-182 cell stemness. These data hint that FOXF1 regulates HCC cell stemness.

\section{Expression of FOXFI affects HCC tumorigenicity and correlates with patient outcome}

A nude mouse xenograft assay was performed to assess the role of FOXF1 in vivo. Our data on tumor size showed that the tumor volume was significantly decreased in FOXF1-overexpressing-cell-injected mice compared with the control mice 4 weeks after injection (Figure 4A). Tumor weights of the mice were measured at the end of the experiment (Figure 4B), and we found that the average tumor weight of the $F O X F 1$-overexpressing xenografts was significantly lower than that of the control xenografts ( $P=0.0023$; Figure 4B). In $F O X F 1$-overexpressing HCC xenografts, the protein levels of CD44 and PCNA, a reported indicator of tumor growth activity in several cancers, ${ }^{21-23}$ were decreased (Figure 4C). All these data suggested that overexpression of FOXF1 inhibited HCC cell tumorigenicity in vivo through decreased cancer cell stemness and inhibition of tumorigenesis.

We next investigated the association between FOXF1 expression and patients' overall survival. Log-rank test showed that patients with high FOXF1 expression level, whose tumor had $>20 \%$ of the nuclei positively stained, had significantly higher survival rates than those patients with low levels of FOXF1 expression ( $P=0.0037$; Figure 4D).

\section{Discussion}

Human HCC accounts for $90 \%$ of cases of primary liver cancer, ${ }^{1}$ and metastasis is one of the most common causes of death among HCC patients. ${ }^{24}$ Although several key factors for HCC are well characterized, the molecular pathogenesis is still not well understood. ${ }^{25,26}$ Mammalian FOXF1 is predominantly expressed in mesenchymal cell types and plays an important role in the generation of mesoderm-derived tissues. ${ }^{27}$ Several publications ${ }^{17,28,29}$ have shown that expression of FOXF1 is downregulated and plays a novel tumor suppressor role in some cases of breast cancer, colorectal cancer, and prostate cancer. However, in lung cancer, overexpression of FOXF1 is linked to cancer-associated stromal fibroblast growth. ${ }^{14}$ Therefore, investigation of expression and function of FOXF1 in HCC cells is important. In this study, we found that the expression level of FOXF1 determined by Q-RTPCR and Western blot was significantly lower in human HCC tissues than in noncancerous adjacent tissues. We also detected FOXF1 expression by immunohistochemistry and found that FOXF1 was mainly localized in the nuclei of HCC cells. Furthermore, the alteration of FOXF1 expression was consistent with the Western blot and Q-RTPCR results. Together, our findings support the 

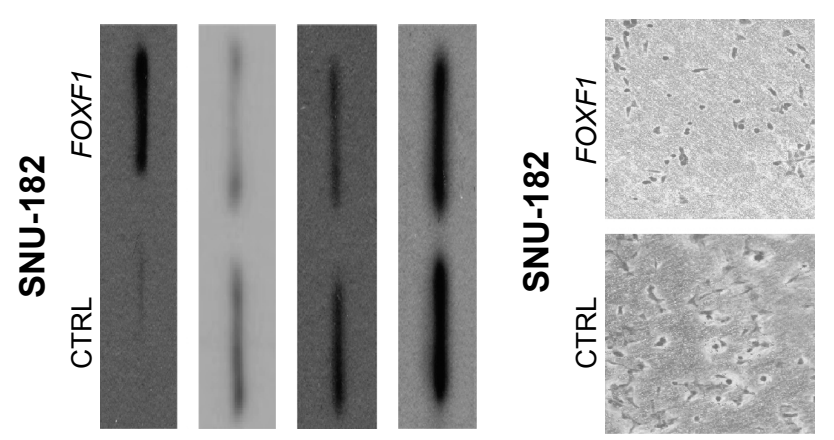
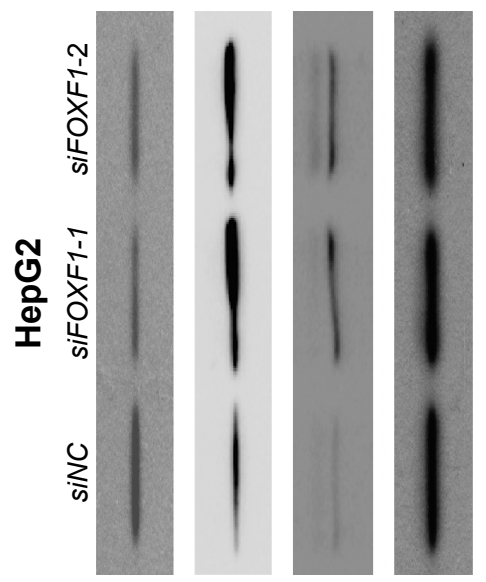

茫

$\mathbf{m}$

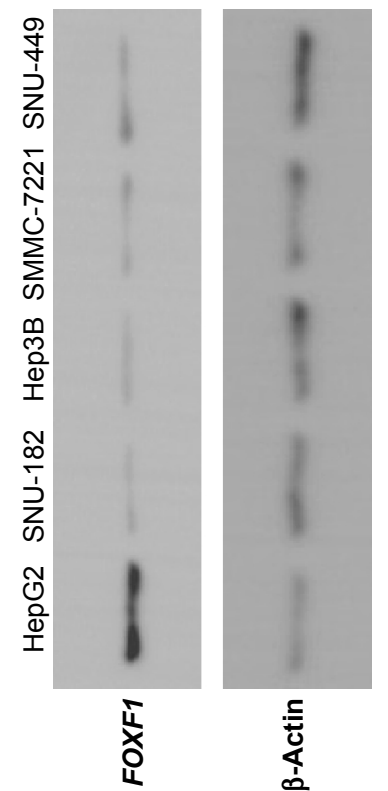

$<$

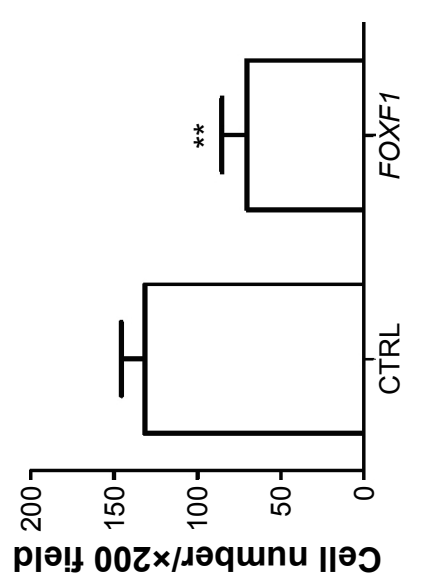

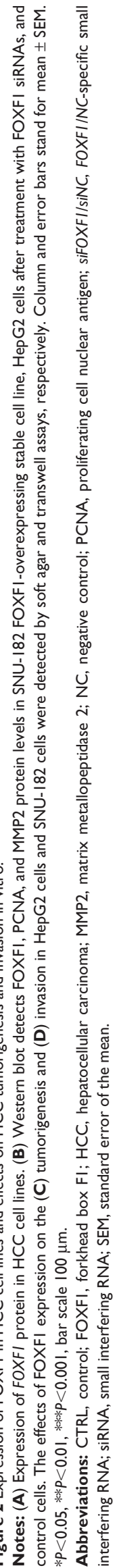


hypothesis that the $F O X F 1$ gene might encode a potentially important protein for HCC progression.

To evaluate the biological effect of FOXF1 on HCC progression, we knocked down the FOXF1 gene in HepG2 cells and overexpressed FOXF1 in SNU-182 cells. Soft agar and transwell assays clearly demonstrated significantly increased in vitro tumorigenesis and invasion abilities in FOXF1-knockdown cells, whereas FOXF1-overexpressed cells had significantly reduced cancer growth and invasion potential. We also performed in vivo study on nude mouse to confirm the effect of FOXF1 overexpression on HCC nude mouse xenografts. Results proved that FOXF1 overexpression could inhibit HCC tumorigenesis in vivo. All these evidences could help us determine the effects of FOXF1 on the biological behavior of HCC cells and offer a new type of targeted therapy.

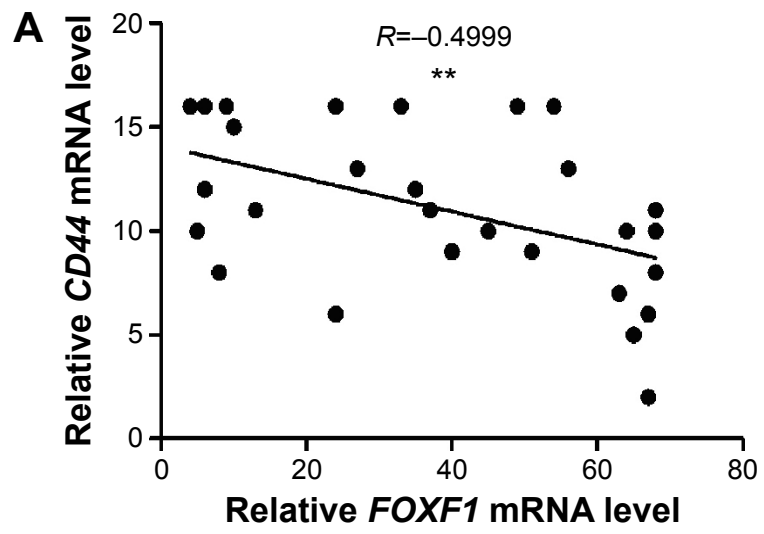

HepG2

B
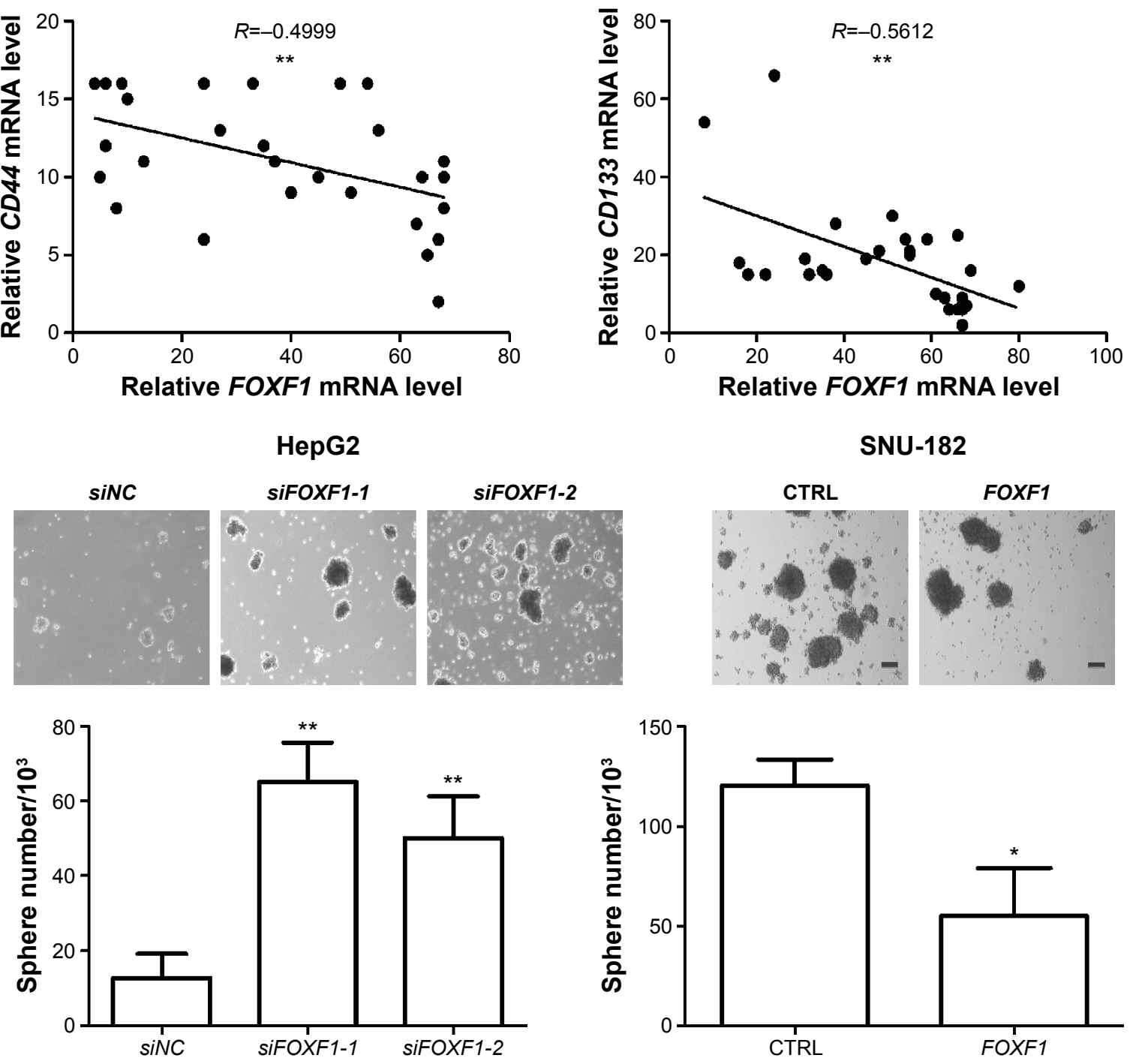

To dissect the potential FOXF1-involving molecular event(s), we screened for the molecular expression of some important signal pathways in HCC specimens (data not shown). The results showed that expression of the HCC stemness marker, CD44, has negative correlation with FOXF1. CD44/133-positive HCC cells were found to have greater migration and stemness abilities; $;^{30-32}$ thus, correlation of FOXF1 expression with CD44 and CD133 expression was analyzed in HCC tumor. Moreover, FOXF1 was also proven to regulate $\mathrm{HCC}$ cell stemness through sphere formation and fluorescence activated cell sorting-based ALDH1- and CD44/133-positive cell counting. In FOXF1-overexpressing xenograft tumors, CD44 also shows downregualtion with decreased tumor size and PCNA level. Moreover, the dysregulation of FOXF1 affects MMP2 protein level, which has been reported to promote HCC invasion and metastasis. ${ }^{33}$
SNU-182
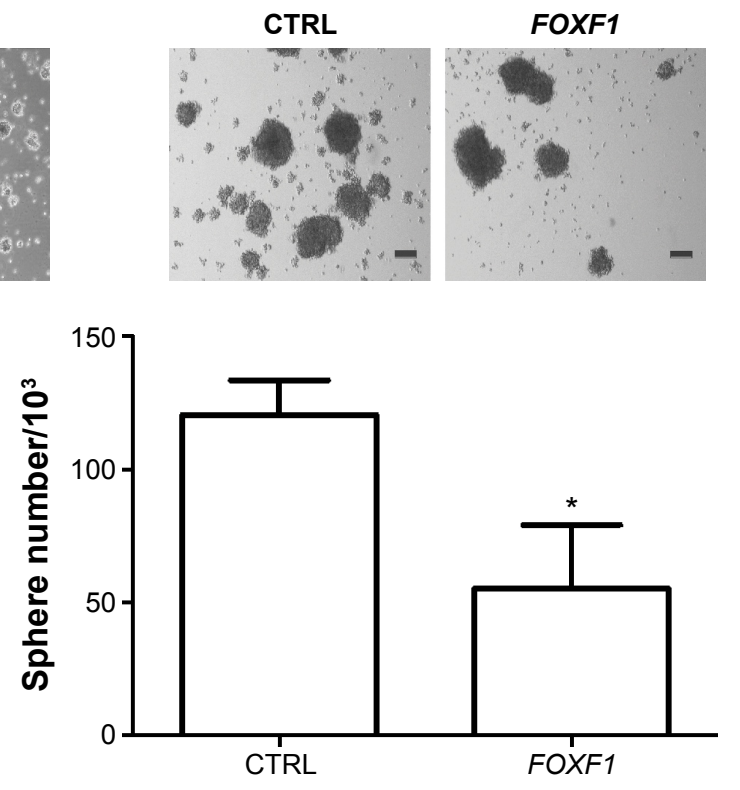

Figure 3 (Continued) 
C

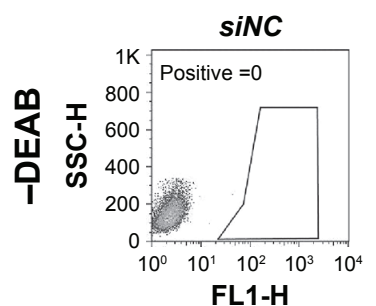

FL1-H

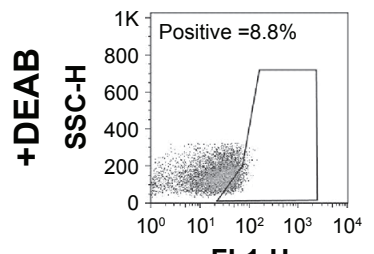

FL1-H
HepG2
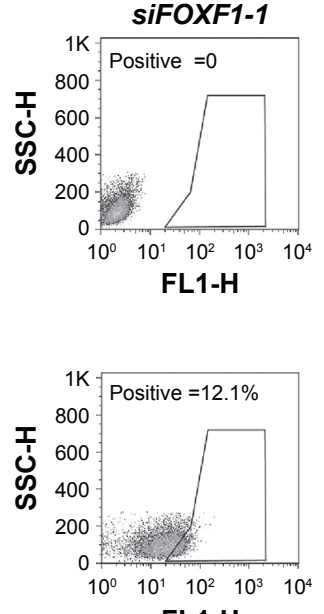

FL1-H
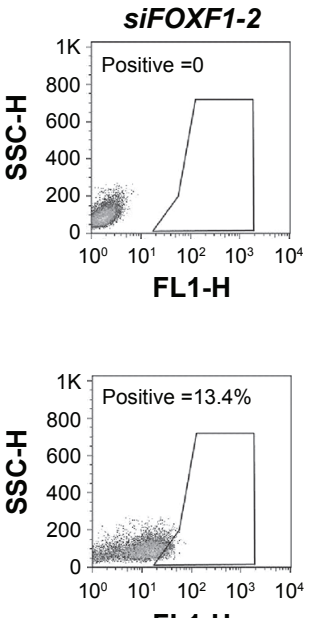

FL1-H
SNU-182
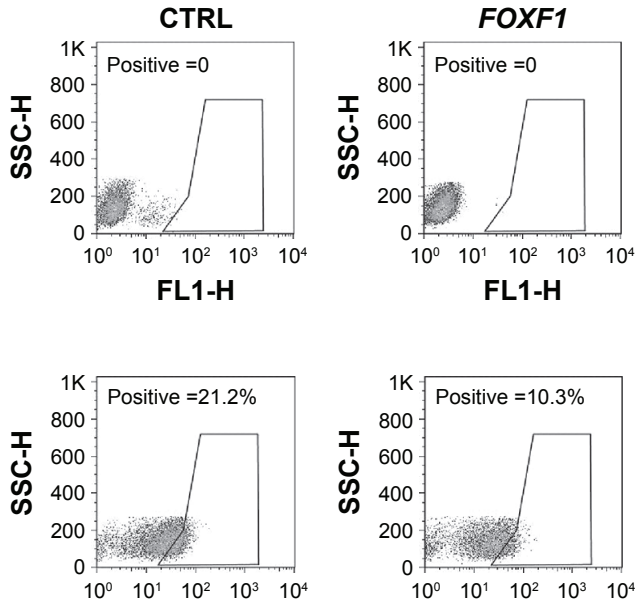

FL1-H

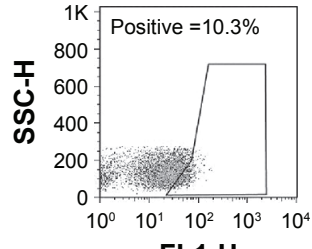

FL1-H
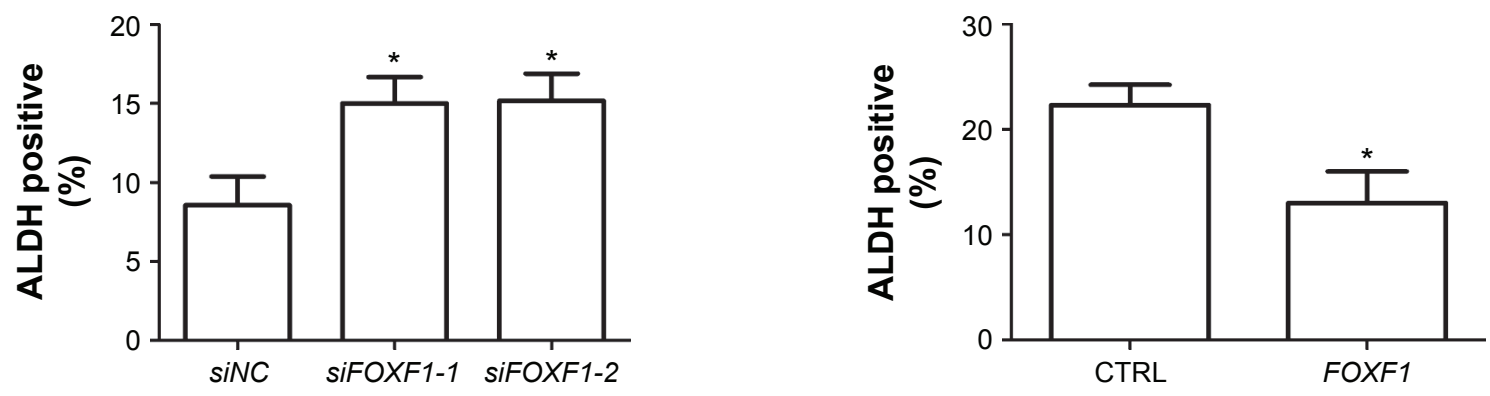

D

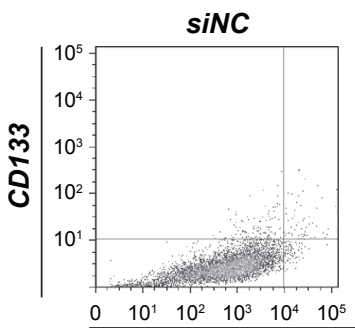

HepG2

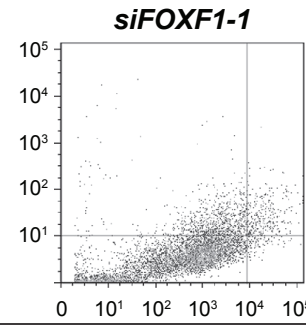

CD44
SNU-182
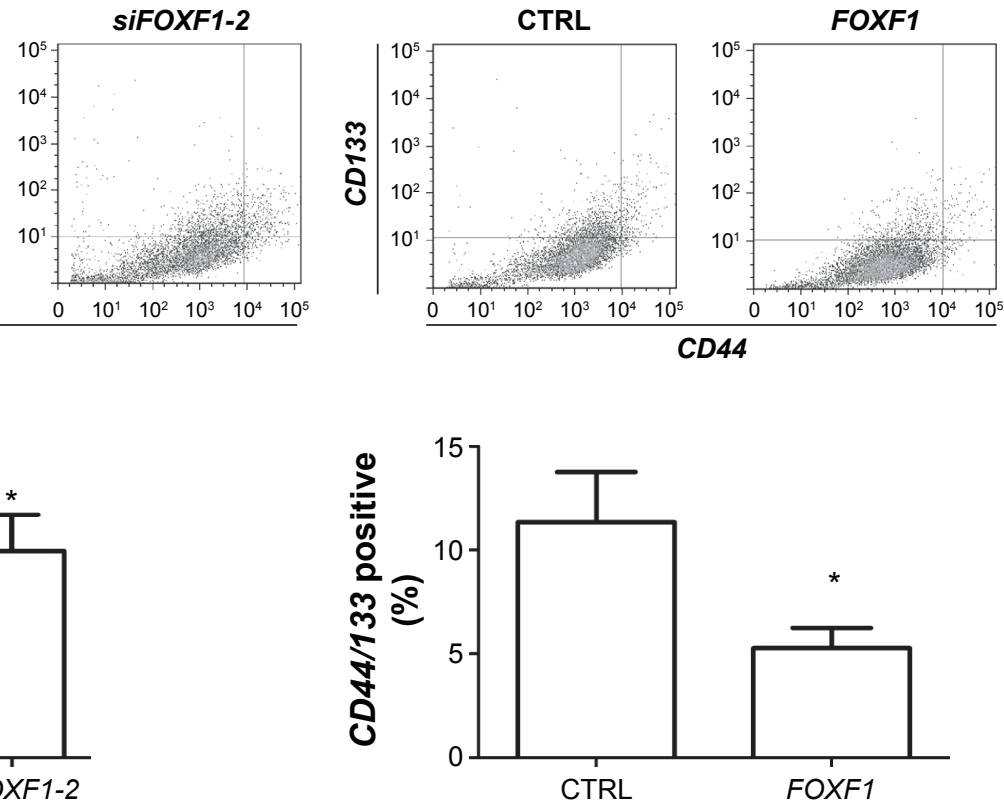

Figure $3 \mathrm{FOXFI}$ affects $\mathrm{HCC}$ cell stemness.

Notes: (A) Correlation of FOXFI mRNA level with CD44 and CDI 33 was analyzed in 30 clinical HCC specimens. HCC cell stemness changes were measured by (B) sphere formation assay, (C) ALDHI, and (D) CD44/I33-positive cell counting when FOXFI expression has been modified. Column and error bars stand for mean \pm SEM. $* P<0.05$, $* * P<0.0$ I, bar scale $190 \mu \mathrm{m}$.

Abbreviations: ALDH, aldehyde dehydrogenase; CTRL, control; DEAB, diethylaminobenzaldehyde; FOXFI, forkhead box FI; HCC, hepatocellular carcinoma; NC, negative control; siFOXFI/siNC, FOXFI/NC-specific small interfering RNA; CD, cluster of differentiation; SEM, standard error of the mean. 


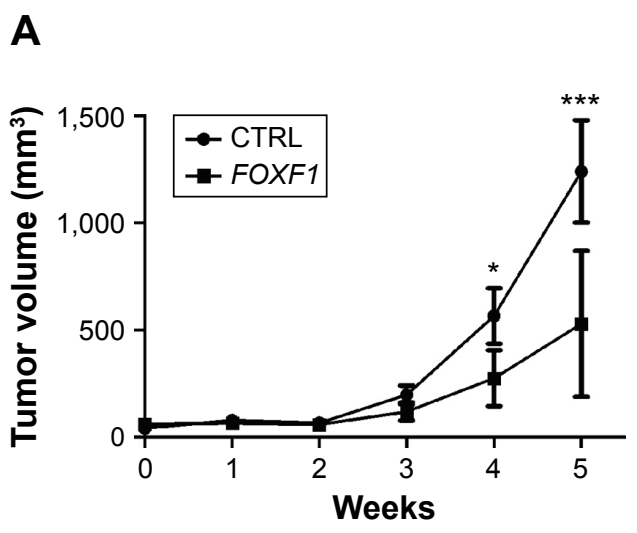

Tumors

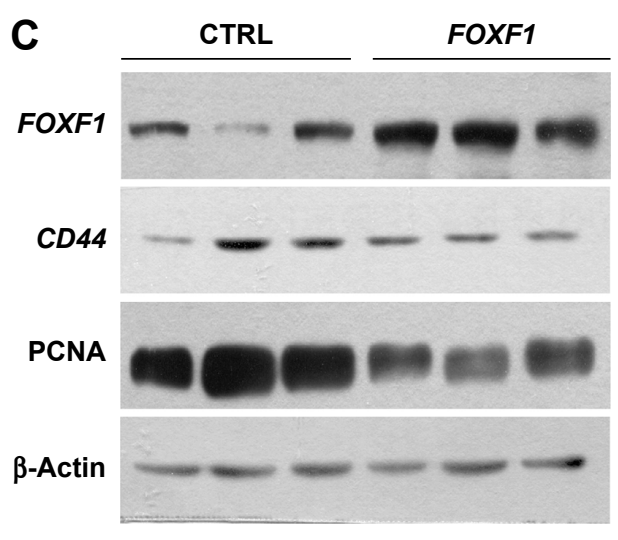

B
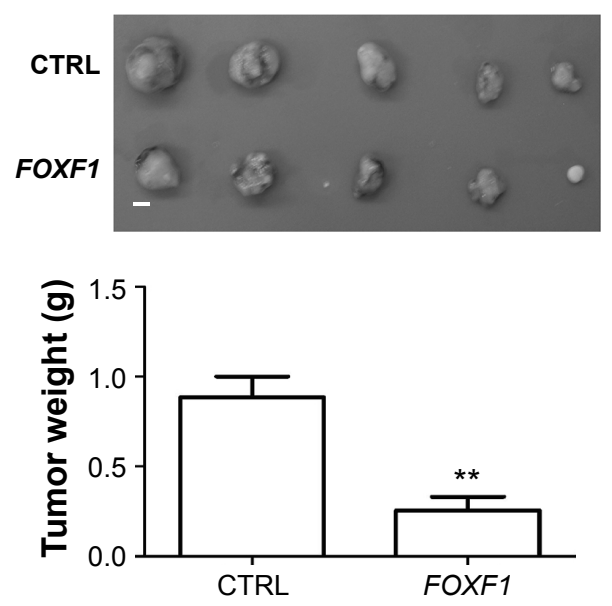

D

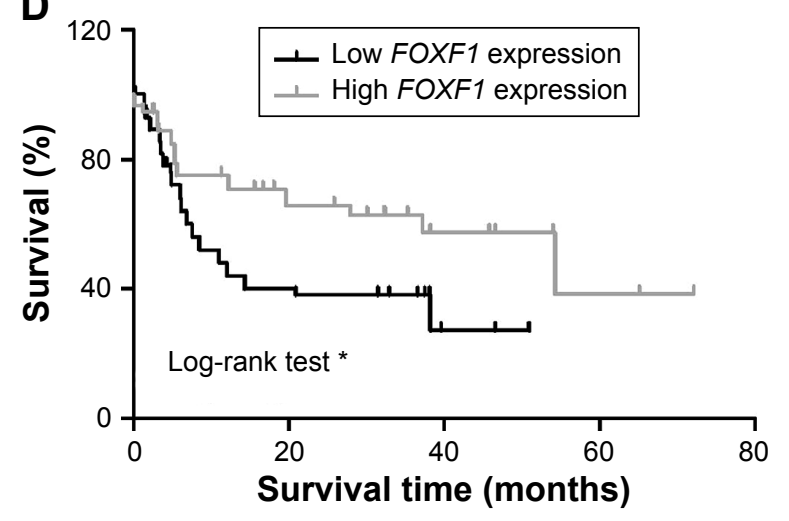

Figure 4 Overexpressed FOXFI inhibits HCC tumorigenicity in nude mice and correlates with better HCC patients' survival.

Notes: (A) In vivo tumor growth curve of FOXFI-overexpressing and control xenografts over 5 weeks. (B) The picture and average tumor weight of the control and FOXFI-overexpressing xenografts at the end point. (C) Western blotting assay of FOXFI, CD44, and PCNA protein expression levels in control and FOXFI-overexpressing xenografts. (D) Relation between FOXFI expression and overall survival in HCC was analyzed by Kaplan-Meier method and the log-rank test. Column and error bars stand for mean \pm SEM. $* P<0.05, * * P<0.01$, $* * * P<0.00$ I, bar scale $5 \mathrm{~mm}$.

Abbreviations: FOXFI, forkhead box FI; HCC, hepatocellular carcinoma; NC, negative control; PCNA, proliferating cell nuclear antigen; siFOXFI/siNC, FOXFI/NC-specific small interfering RNA; SEM, standard error of the mean.

In addition to the functions of FOXF1 in HCC cells, we found that FOXF1 expression remarkably correlates with patients' clinical outcome. High FOXF1 expression group has significantly higher survival rate than the low-expression group. Combined with the results of benchmark tests, low expression of FOXF1 may serve as a poor prognostic factor for HCC patients. Therefore, FOXF1 could be a potential significant biomarker in clinical practice.

\section{Conclusion}

In conclusion, our studies demonstrate that FOXF1 has inhibitory effect on HCC tumorigenesis, invasion, and cell stemness. The FOXF1 expression status is closely correlated with the survival rate of HCC patients. The functions of FOXF1 suggest a key role for FOXF1 in suppressing tumor progression. Therefore, our results suggest that FOXF1 is a novel molecular target that may be potentially used for detecting and treating human HCC.

\section{Acknowledgments}

The study was supported by the Technique Development Foundation and the Young Professionals Foundation of the Affiliated Jiangyin Hospital of Southeast University Medical College (No 201409130017).

\section{Disclosure}

The authors report no conflicts of interest in this work.

\section{References}

1. Jemal A, Bray F, Center MM, Ferlay J, Ward E, Forman D. Global cancer statistics. CA Cancer J Clin. 2011;61(2):69-90.

2. Kohles N, Nagel D, Jungst D, Durner J, Stieber P, Holdenrieder S. Prognostic relevance of oncological serum biomarkers in liver cancer patients undergoing transarterial chemoembolization therapy. Tumour Biol. 2012; 33(1):33-40. 
3. Wang H, Chen L. Tumor microenviroment and hepatocellular carcinoma metastasis. J Gastroenterol Hepatol. 2013;28(suppl 1):43-48.

4. Li J, Yang XM, Wang YH, et al. Monoamine oxidase A suppresses hepatocellular carcinoma metastasis by inhibiting the adrenergic system and its transactivation of EGFR signaling. J Hepatol. 2014;60(6): $1225-1234$.

5. Kim do Y, Paik YH, Ahn SH, et al. PIVKA-II is a useful tumor marker for recurrent hepatocellular carcinoma after surgical resection. Oncology. 2007;72(suppl 1):52-57.

6. Tung-Ping Poon R, Fan ST, Wong J. Risk factors, prevention, and management of postoperative recurrence after resection of hepatocellular carcinoma. Ann Surg. 2000;232(1):10-24.

7. Hannenhalli S, Kaestner KH. The evolution of Fox genes and their role in development and disease. Nat Rev Genet. 2009;10(4):233-240.

8. Katoh M, Katoh M. Human FOX gene family (Review). Int J Oncol. 2004;25(5):1495-1500.

9. Myatt SS, Lam EW. The emerging roles of forkhead box (Fox) proteins in cancer. Nat Rev Cancer. 2007;7(11):847-859.

10. Kaufmann E, Knochel W. Five years on the wings of fork head. Mech Dev. 1996;57(1):3-20.

11. Kalinichenko VV, Bhattacharyya D, Zhou Y, et al. Foxf1 +/- mice exhibit defective stellate cell activation and abnormal liver regeneration following CCl4 injury. Hepatology. 2003;37(1):107-117.

12. Kalinichenko VV, Zhou Y, Bhattacharyya D, et al. Haploinsufficiency of the mouse Forkhead Box f1 gene causes defects in gall bladder development. J Biol Chem. 2002;277(14):12369-12374.

13. Mahlapuu M, Enerback S, Carlsson P. Haploinsufficiency of the forkhead gene Foxf1, a target for sonic hedgehog signaling, causes lung and foregut malformations. Development. 2001;128(12):2397-2406.

14. Saito RA, Micke P, Paulsson J, et al. Forkhead box F1 regulates tumorpromoting properties of cancer-associated fibroblasts in lung cancer. Cancer Res. 2010;70(7):2644-2654.

15. Gialmanidis IP, Bravou V, Petrou I, et al. Expression of Bmi1, FoxF1, Nanog, and gamma-catenin in relation to hedgehog signaling pathway in human non-small-cell lung cancer. Lung. 2013;191(5):511-521.

16. Nilsson J, Helou K, Kovacs A, et al. Nuclear Janus-activated kinase 2/nuclear factor 1-C2 suppresses tumorigenesis and epithelial-tomesenchymal transition by repressing Forkhead box F1. Cancer Res. 2010;70(5):2020-2029.

17. Lo PK, Lee JS, Liang X, et al. Epigenetic inactivation of the potential tumor suppressor gene FOXF1 in breast cancer. Cancer Res. 2010; 70(14):6047-6058.

18. Lo PK, Lee JS, Chen H, Reisman D, Berger FG, Sukumar S. Cytoplasmic mislocalization of overexpressed FOXF1 is associated with the malignancy and metastasis of colorectal adenocarcinomas. Exp Mol Pathol. 2013;94(1):262-269.

19. Chan LH, Luk ST, Ma S. Turning hepatic cancer stem cells inside out - a deeper understanding through multiple perspectives. Mol Cells. 2015;38(3):202-209.
20. Wang Y, Hanifi-Moghaddam P, Hanekamp EE, et al. Progesterone inhibition of Wnt/beta-catenin signaling in normal endometrium and endometrial cancer. Clin Cancer Res. 2009;15(18):5784-5793.

21. Jia W, Lu R, Jia G, Ni M, Xu Z. Expression of pituitary tumor transforming gene (PTTG) in human pituitary macroadenomas. Tumour Biol. 2013;34(3):1559-1567.

22. Ni S, Zhu J, Zhang J, et al. Expression and clinical role of NF45 as a novel cell cycle protein in esophageal squamous cell carcinoma (ESCC). Tumour Biol. 2015;36(2):747-756.

23. Wang Q, Li J, Li G, et al. Prognostic significance of sphingosine kinase 2 expression in non-small cell lung cancer. Tumour Biol. 2014; 35(1):363-368.

24. Lee SC, Tan HT, Chung MC. Prognostic biomarkers for prediction of recurrence of hepatocellular carcinoma: current status and future prospects. World J Gastroenterol. 2014;20(12):3112-3124.

25. Chu R, Mo G, Duan Z, et al. miRNAs affect the development of hepatocellular carcinoma via dysregulation of their biogenesis and expression. Cell Commun Signal. 2014;12:45.

26. Hoshida Y, Nijman SM, Kobayashi M, et al. Integrative transcriptome analysis reveals common molecular subclasses of human hepatocellular carcinoma. Cancer Res. 2009;69(18):7385-7392.

27. Deng J, Fujimoto J, Ye XF, et al. Knockout of the tumor suppressor gene Gprc5a in mice leads to NF-kappaB activation in airway epithelium and promotes lung inflammation and tumorigenesis. Cancer Prev Res. 2010;3(4):424-437.

28. Lo PK, Lee JS, Sukumar S. The p53-p21WAF1 checkpoint pathway plays a protective role in preventing DNA rereplication induced by abrogation of FOXF1 function. Cell Signal. 2012;24(1):316-324.

29. Watson JE, Doggett NA, Albertson DG, et al. Integration of highresolution array comparative genomic hybridization analysis of chromosome $16 \mathrm{q}$ with expression array data refines common regions of loss at 16q23-qter and identifies underlying candidate tumor suppressor genes in prostate cancer. Oncogene. 2004;23(19):3487-3494.

30. Hou Y, Zou Q, Ge R, Shen F, Wang Y. The critical role of CD133(+) $\mathrm{CD} 44(+/$ high) tumor cells in hematogenous metastasis of liver cancers. Cell Res. 2012;22(1):259-272.

31. Yamashita T, Kaneko S. Orchestration of hepatocellular carcinoma development by diverse liver cancer stem cells. J Gastroenterol. 2014; 49(7):1105-1110.

32. Yamashita T, Wang XW. Cancer stem cells in the development of liver cancer. J Clin Invest. 2013;123(5):1911-1918.

33. Wang B, Ding YM, Fan P, Wang B, Xu JH, Wang WX. Expression and significance of MMP2 and HIF-1alpha in hepatocellular carcinoma. Oncol Lett. 2014;8(2):539-546.
OncoTargets and Therapy

\section{Publish your work in this journal}

OncoTargets and Therapy is an international, peer-reviewed, open access journal focusing on the pathological basis of all cancers, potential targets for therapy and treatment protocols employed to improve the management of cancer patients. The journal also focuses on the impact of management programs and new therapeutic agents and protocols on

\section{Dovepress}

patient perspectives such as quality of life, adherence and satisfaction The manuscript management system is completely online and includes a very quick and fair peer-review system, which is all easy to use. Visit $\mathrm{http}: / /$ www.dovepress.com/testimonials.php to read real quotes from published authors. 